\title{
COMUNICAÇÃO
}

\section{PROCESSAMENTO DE CONSERVAS DE PALMITO CAULINAR DE PUPUNHA CONTENDO DIFERENTES GRAUS DE ACIDEZ}

\author{
Processing of canned stalk-of-palm containing different degrees of acidity \\ Marcelo Gomes ${ }^{1}$, Janaina do Valle ${ }^{1}$, Dorivaldo da Silva Raupp 2 , Francisco Paulo Chaimsohn 3 , \\ Aurélio Vinicius Borsato ${ }^{4}$
}

\begin{abstract}
RESUMO
Uma das vantagens da pupunha (Bactris gasipaes) para produção de palmito é seu rendimento, relativamente alto, de palmito caulinar, que pode ser processado de diversas formas. O procedimento de acidificação é essencial para a qualidade dessas conservas no sentido de resultar produtos seguros para o consumo. A pesquisa teve por proposta determinar a suficiência do procedimento de acidificação, aplicado ao processamento de conservas de palmito caulinar para abaixar o $\mathrm{pH}$ do palmito in natura até pH seguro dentro das conservas, que, segundo a norma oficial, deve permanecer no equilíbrio igual ou abaixo de 4,5. O processamento que projetou uma acidificação para produzir no equilíbrio $\mathrm{pH}$ 4,3 resultou em algumas conservas contendo valores de $\mathrm{pH}$ superiores a 4,5, portanto, em desacordo com a norma oficial. No entanto, nos processamentos em que foi projetado para o equilíbrio o pH 4,2 ou 3,9, todas as conservas foram seguras para o consumo. Assim, foi concluído que, para resultar em segurança alimentar, a execução do procedimento de acidificação deve projetar para o pH de equilíbrio um valor abaixo de 4,3, independentemente da salmoura escolhida para a conserva.
\end{abstract}

Termos para indexação: Bactris gasipaes, palmito basal de pupunha, acidificação de conservas, qualidade, segurança alimentar.

\begin{abstract}
One of the advantages of pupunha (Bactris gasipaes) for the production of heart of palm is a relatively high heart of palm yield obtained from stems, which can be processed in a number of ways. The acidification procedure is essential for the quality of the canned when it comes to yielding products that are safe for consumption. This research aimed to determine whether the acidification procedure, when applied to the processing of canned stalk-of-palm, is sufficient to lower the $\mathrm{pH}$ value of fresh stalk-of-palm to a safe value, which, according to official regulations, should remain equal to or below 4.5 when in equilibrium. The processing that projected an acidification that would produce a $\mathrm{pH}$ value of 4.3 when in equilibrium resulted in some canned containing $\mathrm{pH}$ values higher than 4.5 , therefore not complying with the official regulation. However, in processings where $\mathrm{pH}$ values of 4.2 or 3.9 at equilibrium were projected, all canned were safe for consumption. Therefore, it was concluded that for an acidification procedure that will result in consumption safety to be carried out, a pH value below 4.3 at equilibrium must be projected, regardless of the brine selected for preservation.
\end{abstract}

Index terms: Bactris gasipaes, stalk-of-palm, acidity of canned, quality, food safety.

(Recebido para publicação em 3 de janeiro de 2005 e aprovado em 6 junho de 2005)

O palmito de pupunha é o resultado da domesticação de várias espécies silvestres, encontradas desde a Bolívia até a Nicarágua, as quais têm sofrido múltiplas hibridações e resultaram numa "espécie sintética”, a Bactris gasipaes H.B.K. (IAPAR, 2001).

Apesar do sabor ligeiramente adocicado e da coloração levemente amarelada (creme), características que distingüem a pupunha das demais espécies produtoras de palmito, a não ocorrência de escurecimento enzimático durante o processamento, bem como a sua textura macia (BERNHARDT, 1999; FERREIRA et al., 1982; SOARES, 1997) são outras características vantajosas para a produção de conservas de palmito, com aproveitamento máximo tanto da matéria-prima foliar como da caulinar (basal). Ainda, também por isso, constitui-se em matéria-prima propícia para a comercialização como produto fresco (KAPP et al., 2003).

O palmito é constituído de três partes (RAUPP \& CHAIMSOHN, 2001): caulinar (basal), de maior diâmetro, situada na região mais baixa do talo do palmito; apical, de aspecto foliar e diâmetro reduzido, situada no ápice do talo; e, creme, coração ou tolete, localizada entre as partes basal e apical. Uma das vantagens da pupunha é o rendimento, relativamente alto, de palmito caulinar que se constitui em um produto para elaboração de diversos tipos de conserva.

\footnotetext{
${ }^{1}$ Graduando(a) da Universidade Estadual de Ponta Grossa - DEA-Laboratório de Tecnologia de Alimentos, F-29/Agronomia - Ponta Grossa, PR. ${ }^{2}$ Professor Pesquisador - Universidade Estadual de Ponta Grossa - DEA-Laboratório de Tecnologia de Alimentos, F-29/Agronomia Campus Uvaranas - Av. Carlos Cavalcanti, 4748 - 84030-900 - Ponta Grossa, PR - raupp@uepg.br

${ }^{3}$ Pesquisador, Instituto Agronômico do Paraná/IAPAR-PG - Ponta Grossa, PR.

${ }^{4}$ Doutorando em Agronomia - Universidade Federal do Paraná - Curitiba, PR.
} 
O Clostridium botulinum é uma bactéria grampositiva, anaeróbia obrigatória, que se desenvolve geralmente em alimentos de $\mathrm{pH}$ superior a 4,6, classificados como pouco ácidos, (FRANCO \& LANDGRAF, 1996; MONTES, 1977; PELCZAR, 1981; RIEDEL, 1992) que foram inadequadamente envasados (PASCHOALINO, 1994; RAUPP \& CHAIMSOHN, 2001). O palmito de pupunha apresenta $\mathrm{pH}$ natural superior a 4,5, freqüentemente entre 5,6 e 6,2 (CHAIMSOHN, 2000; RAUPP \& CHAIMSOHN, 2001; TONET et al., 1999; ZAPATA \& QUAST, 1975). Quando, durante o processamento de conservas, as características desse produto permanecem favoráveis ao desenvolvimento do $C$. botulinum, como $\mathrm{pH}$ do palmito superior a 4,5, os esporos dessa bactéria termo-resistentes ao trata-mento térmico aplicado podem se desenvolver para as formas vegetativas (PASCHOALINO, 1997; RAUPP \& CHAIMSOHN, 2001), e, por conseguinte, produzem uma toxina que causa a síndrome conhecida como botulismo, a qual, não raramente, pode resultar em óbito para o consumidor que ingerir o produto alimentício contendo tal toxina (BRIMELOW \& VADEHRA, 1992; CAMARGO, 1984; FELLOWS, 1994; GAVA, 1978).

A segurança alimentar dessas conservas é obtida combinando-se procedimentos de tratamento térmico e acidificação. Um procedimento de acidificação bem sucedido deve, portanto, resultar em um $\mathrm{pH}$ no produto abaixo ou igual a 4,5, segundo a norma oficial (BRASIL, 1999), além de permitir o uso de um tratamento térmico abrandado, de modo a não danificar a textura agradável do palmito (FRANCO \& LANDGRAF, 1996; LINDEN \& LORIENT, 1996; PASCHOALINO, 1994, 1997; RAUPP, 2002; RAUPP \& CHAIMSOHN, 2001).

A pesquisa teve por proposta avaliar a suficiência do procedimento de acidificação aplicado ao processamento de conservas de palmito caulinar (basal) de pupunha, para abaixar o $\mathrm{pH}$ do palmito in natura até $\mathrm{pH}$ seguro dentro das conservas, que, segundo a norma oficial, deve permanecer no equilíbrio igual ou abaixo de 4,5, usando o ácido cítrico como ingrediente acidificante.

O palmito pupunha usado na pesquisa, foi cultivado na Estação Experimental de Morretes I - Instituto Agronômico do Paraná - IAPAR, em Morretes-PR, Brasil $\left(25^{\circ} 30^{\prime}\right.$ latitude $\mathrm{S}, 48^{\circ} 49^{\prime}$ longitude $\mathrm{W}$, altitude $59 \mathrm{~m}$ e clima Af). Os talos permaneceram na temperatura ambiente e em local apropriado por cerca de dois dias até o processamento. Os materiais e instalações, como facas, mesa inox, recipientes, foram devidamente higienizados seguindo as recomendações de Raupp \& Chaimsohn (2001).
Foram executados três experimentos, sendo que cada experimento foi constituído de três tratamentos e dez observações/tratamento. A norma oficial (BRASIL, 1999) considera que o $\mathrm{pH}$ de uma conserva de palmito deve permanecer, no equilíbrio, igual ou abaixo de 4,5, independentemente do tempo de consumo dentro do período de validade, por isso as dez observações do tratamento avaliadas em tempos diferentes foram consideradas como repetições. Cerca de 130 talos de palmito foram processados para o envase de 90 vidros de conservas de palmito caulinar (basal) de pupunha. Depois de removidas as capas externas, foram preparados cortes da parte caulinar em rodelas medindo cerca de 7-10 $\mathrm{mm}$ de espessura, as quais foram, a seguir, cortadas em formato triangular e mantidas em água potável, até o envase.

No primeiro experimento, o procedimento de acidificação aplicado durante o processamento projetou pH 4,3 para o equilíbrio das conservas. As 30 unidades de conservas constituíram três tratamentos, sendo $10 \mathrm{com}$ salmoura de $20 \mathrm{~g} \mathrm{~L}^{-1}$ de $\mathrm{NaCl}$ (Tratamento 1), $10 \mathrm{com}$ salmoura de $30 \mathrm{~g} \mathrm{~L}^{-1}$ de $\mathrm{NaCl}$ (Tratamento 2) e $10 \mathrm{com}$ salmoura de $40 \mathrm{~g} \mathrm{~L}^{-1}$ de $\mathrm{NaCl}$ (Tratamento 3). No segundo experimento, o procedimento de acidificação aplicado durante o processamento projetou $\mathrm{pH}$ 4,2 para o equilíbrio das conservas. As 30 unidades de conservas constituíram três tratamentos, sendo $10 \mathrm{com}$ salmoura de $20 \mathrm{~g} \mathrm{~L}^{-1} \mathrm{de}$ $\mathrm{NaCl}$ (Tratamento 1), 10 com salmoura de $30 \mathrm{~g} \mathrm{~L}^{-1} \mathrm{de} \mathrm{NaCl}$ (Tratamento 2) e $10 \mathrm{com}$ salmoura de $40 \mathrm{~g} \mathrm{~L}^{-1} \mathrm{de} \mathrm{NaCl}$ (Tratamento 3). No terceiro experimento, o procedimento de acidificação aplicado durante o processamento projetou pH 3,9 para o equilíbrio das conservas. As 30 unidades de conservas constituíram três tratamentos, sendo $10 \mathrm{com}$ salmoura de $20 \mathrm{~g} \mathrm{~L}^{-1}$ de $\mathrm{NaCl}$ (Tratamento 1), $10 \mathrm{com}$ salmoura de $30 \mathrm{~g} \mathrm{~L}^{-1}$ de $\mathrm{NaCl}$ (Tratamento 2) e $10 \mathrm{com}$ salmoura de $40 \mathrm{~g} \mathrm{~L}^{-1}$ de $\mathrm{NaCl}$ (Tratamento 3).

As salmouras contendo $30 \mathrm{~g} \mathrm{~L}^{-1}$ e $40 \mathrm{~g} \mathrm{~L}^{-1}$ são comumente aplicadas pelas agroindústrias no processamento de conservas de palmito, por isso foram usadas na pesquisa. Salmouras contendo menor concentração de sal não costumam ser aplicadas para as conservas de pupunha porque nelas ainda perduram o sabor adocicado e característico do palmito de pupunha. Apesar disso, a salmoura de $20 \mathrm{~g} \mathrm{~L}^{-1}$ foi incluída para comparação.

O procedimento de acidificação das conservas consistiu em acrescentar à salmoura, com diferentes concentrações de sal (NaCl): $20 \mathrm{~g} \mathrm{~L}^{-1}, 30 \mathrm{~g} \mathrm{~L}^{-1}$ ou $40 \mathrm{~g} \mathrm{~L}^{-1}$, o agente acidificante (ácido cítrico), cuja concentração foi determinada como descrito a seguir: foi pesado, para cada

Ciênc. agrotec., Lavras, v. 30, n. 3, p. 569-574, maio/jun., 2006 
experimento, uma amostra de $100 \mathrm{~g}$ de palmito misturada com água potável fornecida pela rede pública (mantendo a proporção média das unidades de envase) e o conteúdo foi triturado em liquidificador industrial para transformação numa massa de palmito. A massa obtida, depois de determinado o seu $\mathrm{pH}$, foi titulada com solução aquosa contendo $50 \mathrm{~g} \mathrm{~L}^{-1}$, do agente acidificante, o ácido cítrico, até: $\mathrm{pH}$ 4,3 para o experimento 1; 4,2 para o experimento 2; e 3,9 para o experimento 3. A proporção média de palmito caulinar e salmoura nas embalagens, em peso, considerada para o procedimento de acidificação foi determinada durante o envase e foi, respectivamente, igual a: $279,3 \mathrm{~g}$ e $281,6 \mathrm{~g}$ para o experimento $1 ; 289,7 \mathrm{~g}$ e $261,6 \mathrm{~g}$ para o experimento $2 ; 323,8 \mathrm{~g}$ e 239,4 g para o experimento 3. Com base no dado do procedimento de titulação - até $\mathrm{pH} 4,3$; 4,2; 3,9 - e conhecidos os pesos médios do palmito e da salmoura nas embalagens, foi determinada a concentração de ácido cítrico da salmoura, usando a fórmula de Zapata \& Quast (1975):

$$
\mathrm{Cs}(\mathrm{g} \mathrm{L}-1)=\mathrm{Cp} \times(\mathrm{Mp} / \mathrm{Ms})
$$

$\mathrm{C}_{\mathrm{s}}=$ concentração de ácido cítrico na salmoura, em $\mathrm{g}$ de ácido cítrico por $100 \mathrm{~g}$ de salmoura, determinada por aplicação da fórmula;

$\mathrm{C}_{\mathrm{p}}=$ concentração de ácido cítrico no palmito basal, em $\mathrm{g}$ de ácido cítrico por $100 \mathrm{~g}$ de palmito, determinada durante a titulação do palmito;

$\mathrm{M}_{\mathrm{p}}=$ peso médio de palmito basal, contido nas embalagens de conserva, em $\mathrm{g}$;

$\mathrm{M}_{\mathrm{s}}=$ peso médio de salmoura, contida nas embalagens de conserva, em $\mathrm{g}$.

As embalagens contendo o produto foram hermeticamente fechadas, tratadas termicamente em temperatura de fervura por 70 minutos e resfriadas com água potável. A seguir, foram identificadas e guardadas em temperatura ambiente até análise.

A estabilização da acidez das conservas foi monitorada periodicamente e através da análise de determinação do $\mathrm{pH}$, como descrito nas normas técnicas do Instituto Adolfo Lutz (1985), e executada em duplicata em uma unidade de embalagem de cada período e de cada lote (tratamento) de 10 unidades (observações) de conservas. Para a medida do $\mathrm{pH}$ do palmito, foi recolhida uma porção de palmito drenado, triturado até produzir uma massa e, a seguir, foi feita a determinação (em duplicata) diretamente nessa massa. Quanto à determinação do $\mathrm{pH}$ da salmoura, a leitura (em duplicata) foi direta no próprio conteúdo da embalagem.
Os dados referentes ao $\mathrm{pH}$ das conservas de palmito caulinar de pupunha foram avaliados usando a análise descritiva e através de gráficos, tendo por propósito comparar o pH de equilíbrio pós-processamento determinado nas unidades de embalagens (dez observações) com o $\mathrm{pH}$ projetado durante o processamento (acidificação), bem como com o pH exigido pela norma oficial (BRASIL, 1999), segundo a qual, a acidificação deve produzir pH abaixo ou igual a 4,5. Portanto, o tratamento aplicado foi considerado seguro somente quando os valores para o $\mathrm{pH}$ de todas as unidades (dez) de embalagens do tratamento satisfizeram, independentemente do período da análise, às exigências da norma oficial.

Os resultados do $\mathrm{pH}$ de equilíbrio para as conservas de palmito pupunha caulinar, os quais avaliaram a eficiência do procedimento de acidificação aplicado durante os processamentos, são apresentados nas Figuras de 1 a 3. O procedimento de acidificação das conservas com o ácido cítrico, como esperado, resultou em diminuição no $\mathrm{pH}$ do palmito in natura, que se situou entre 5,93 a 5,82 .

O sabor doce característico do palmito pupunha não foi percebido na degustação das conservas contendo a salmoura de $30 \mathrm{~g} \mathrm{~L}^{-1}$ ou $40 \mathrm{~g} \mathrm{~L}^{-1}$. Em contraste, os palmitos das conservas contendo a salmoura de $20 \mathrm{~g} \mathrm{~L}^{-1}$ apresentaram ainda um sabor doce residual.

Os valores de $\mathrm{pH}$ no equilíbrio, tanto do palmito como da salmoura, não alcançaram exatamente as projeções feitas durante o processamento, para quaisquer dos tratamentos de acidificação (Figuras 1, 2 e 3).

Apenas os tratamentos de acidificação dos dois processamentos cujos valores de $\mathrm{pH}$ foram estimados para atingir no equilíbrio, respectivamente 4,2 (Figura 2) e 3,9 (Figura 3), produziram conservas de palmito pupunha caulinar consideradas seguras para consumo, de acordo com a norma oficial brasileira (BRASIL, 1999), a qual determina o valor igual ou abaixo de $\mathrm{pH} 4,5$ para esse produto. Esses produtos apresentaram $\mathrm{pH}$ considerados seguros já no $1^{\circ}$ dia depois de processados e se mantiveram no patamar de segurança alimentar durante todo o período de vida de prateleira, cerca de 1,5 anos.

Em contraste, várias observações dos tratamentos em que o $\mathrm{pH}$ foi estimado no processamento para 4,3 (Figura 1), e cujas análises foram feitas também em períodos distintos, apresentaram valores de $\mathrm{pH}$ no equilíbrio superiores ao limite oficial, sendo, por isso, consideradas impróprias para o consumo. 

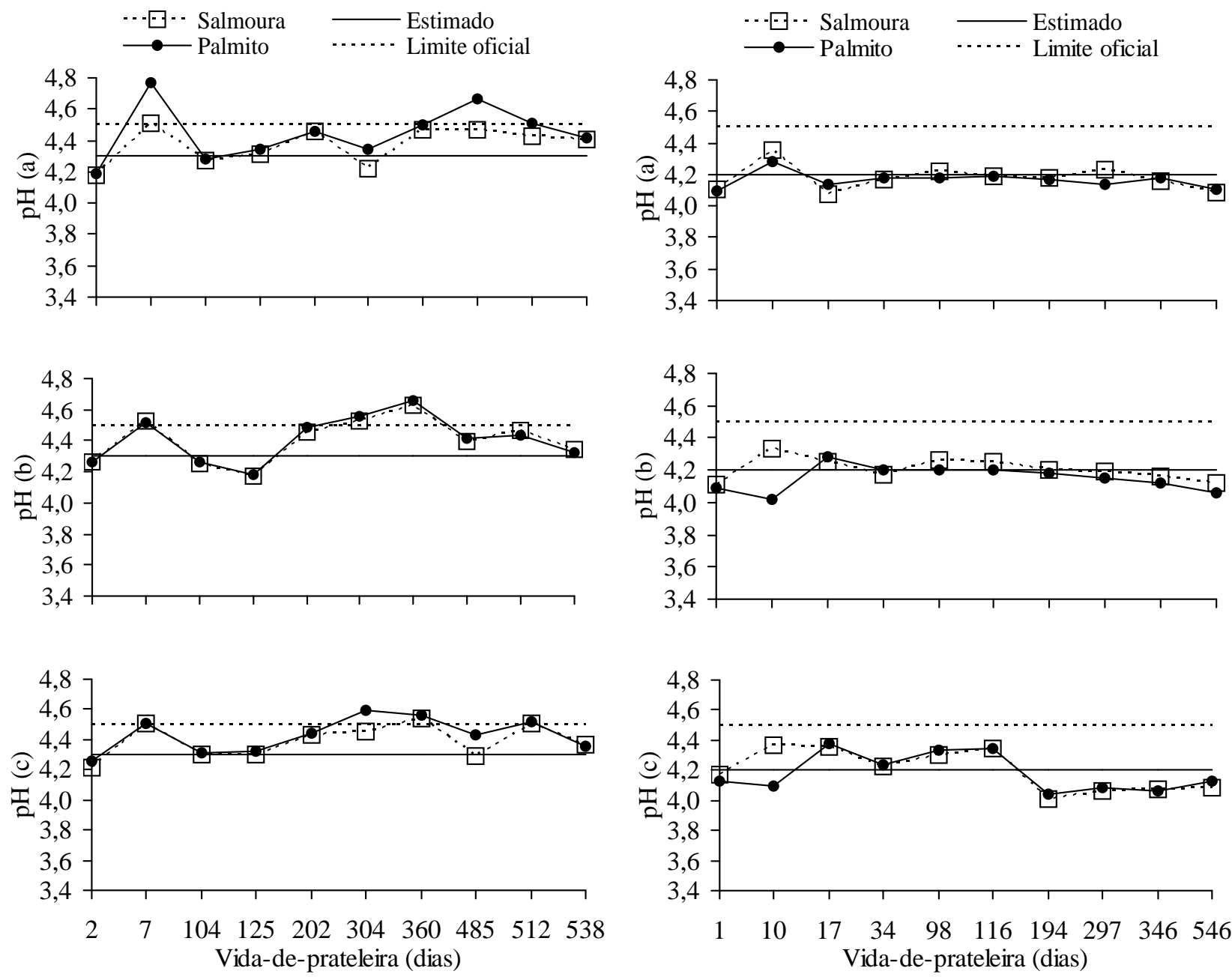

FIGURA 1 - Valores de $\mathrm{pH}$ das conservas de tratamentos contendo salmoura de $20 \mathrm{~g} \mathrm{~L}^{-1}$ (a), $30 \mathrm{~g} \mathrm{~L}^{-1}$ (b) e $40 \mathrm{~g} \mathrm{~L}^{-1}$ (c) e com $\mathrm{pH}$ projetado no procedimento de acidificação do processamento para 4,3 .

Aparentemente nenhuma das três salmouras influenciou de forma marcante no $\mathrm{pH}$ de equilíbrio das conservas para qualquer dos valores projetados para o $\mathrm{pH}$, durante o procedimento de acidificação, respectivamente iguais a 4,3-4,2-3,9.

Diferenças na proporção, em peso, do conteúdo de palmito e de salmoura determinaram as variações do $\mathrm{pH}$ de equilíbrio entre unidades de embalagens (observações/repetições) de cada tratamento. Apesar do

rigor aplicado, o fatiamento do palmito em formato triangular e a arrumação das fatias durante o envase não foi uniforme, por isso, se constituem em causas importantes para as variações encontradas nos pesos do conteúdo de palmito e de salmoura entre unidades de embalagens. Também, o procedimento de acidificação leva em conta os pesos médios de palmito e de salmoura das unidades envasadas e não os pesos por unidade de embalagem.

Ciênc. agrotec., Lavras, v. 30, n. 3, p. 569-574, maio/jun., 2006 

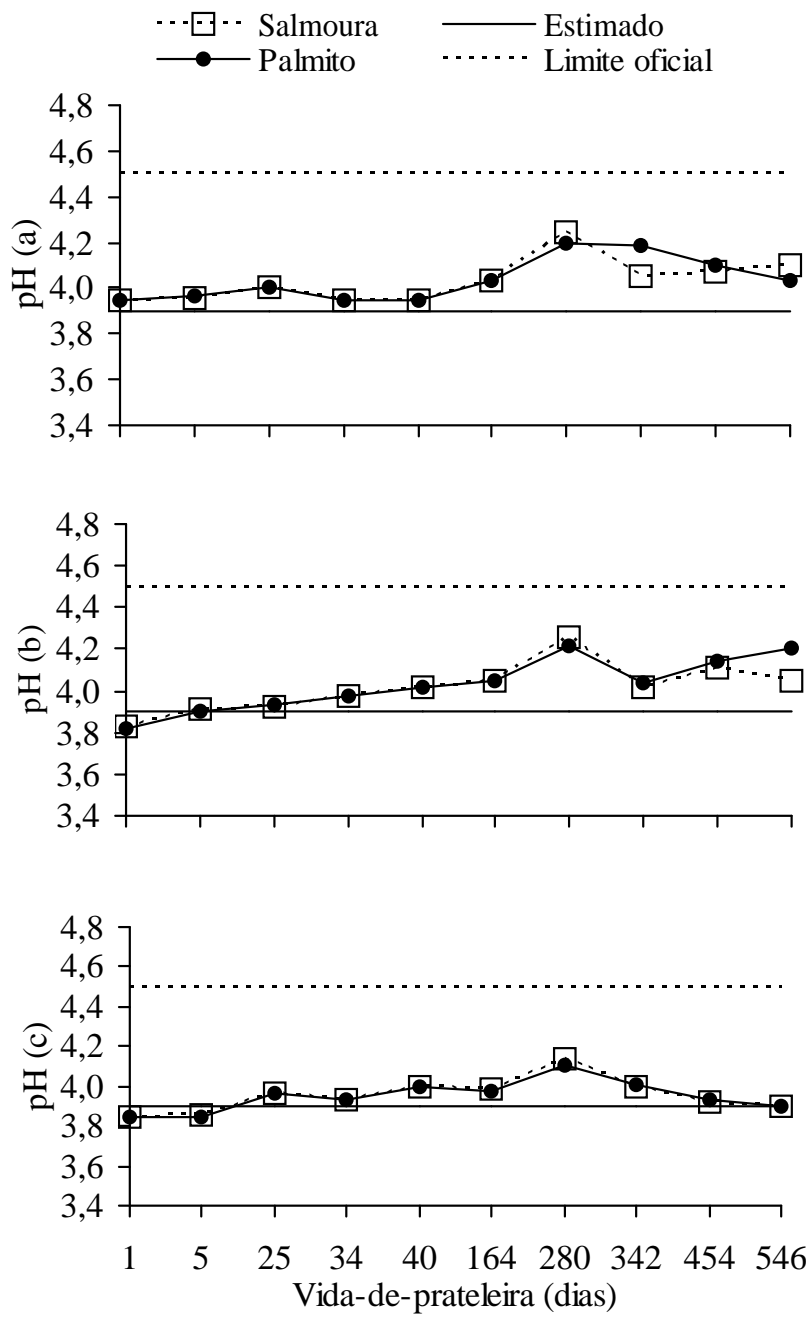

FIGURA 3 - Valores de $\mathrm{pH}$ das conservas de tratamentos contendo salmoura de $20 \mathrm{~g} \mathrm{~L}^{-1}$ (a), $30 \mathrm{~g} \mathrm{~L}^{-1}$ (b) e $40 \mathrm{~g} \mathrm{~L}^{-1}$ (c) e com $\mathrm{pH}$ projetado no procedimento de acidificação do processamento para 3,9.

Segundo Raupp \& Chaimsohn (2001), quanto maior o excesso de peso de palmito dentro da embalagem em relação ao peso médio considerado para o cálculo da estimativa de $\mathrm{pH}$, maior é a possibilidade da conserva apresentar um valor para o $\mathrm{pH}$ considerado de risco, por conseguinte que possibilite o desenvolvimento do $C$. botulinum. Em contrapartida, quando o peso do palmito está abaixo do valor médio estimado, não há riscos para a saúde do consumidor, porque, nessas condições, a unidade de embalagem recebe, por conter mais salmoura, uma quantidade de ácido cítrico superior à requerida.
Fatores relacionados com a execução do procedimento analítico de determinação do $\mathrm{pH}$ de equilíbrio, como a amostragem, a drenagem da amostra de palmito para o escoamento da salmoura, o grau de trituração do palmito, também podem ter contribuído para as variações do $\mathrm{pH}$ de equilíbrio entre as unidades de conservas do tratamento. E, uma possível degradação do agente acidificante, devido à ação do tratamento térmico, também não pode ser descartada como um outro fator de interferência.

Há outros fatores que poderiam influir no $\mathrm{pH}$ de equilíbrio da conserva, que são corrigidos pelo procedimento de acidificação. Entre os quais pode-se mencionar: (a) diferenças no grau de acidez do palmito caulinar entre regiões edafoclimáticas distintas (CHAIMSOHN, 2002); (b) umidade do solo no período de colheita; (c) grau de pureza do agente acidificante; e, aparentemente, (d) a idade do palmito.

Considerando-se que o controle de qualidade do processamento deve ter por meta uma produção sem ocorrência de pH acima de 4,5, bem como a existência de fatores que interferem, apesar do rigor aplicado, na acidez projetada durante o processamento para a conserva, é prudente recomendar procedimentos de acidificação que projetem valores de equilíbrio de $\mathrm{pH}$ igual ou abaixo de 4,2 . $\mathrm{O}$ atributo sensorial paladar poderá ser, então, o fator limitador para usos de pH muito abaixo de 4,2.

$\mathrm{O}$ procedimento de acidificação que projetou durante o processamento das conservas de palmito caulinar de pupunha um $\mathrm{pH}$ de equilíbrio igual a $4,2 \mathrm{ou}$ 3,9 se mostrou, contrastando com o procedimento de $\mathrm{pH} 4,3$, suficiente para produzir produtos seguros, de acordo com a norma oficial, para o consumo na alimentação humana.

Os autores agradecem ao Programa PRONAF, por ter financiado o projeto "Avaliação e desenvolvimento de tecnologias e sistemas de cultivo para viabilização da produção de palmito de pupunha (Bactris gasipaes) e palmeira-real-da-austrália (Archontophoenix alexandrae H. Wendl. \& Drude) para diversificação de sistemas de produção familiares do Paraná", sob a coordenação do Instituto Agronômico do Paraná - IAPAR e ao CNPq pelo fornecimento de uma bolsa de iniciação científica do Programa PIBIC-Institucional.

\section{REFERÊNCIAS BIBLIOGRÁFICAS}

RNHART, L. W. Características do palmito da pupunheira do ponto de vista do processamento. Documentos, Porto Velho, v. 41, p. 24-33, 1999. 
BRIMELOW, C.; VADEHRA, D. Refrigeración. In: ARTHEY, D.; DENNIS, C. Procesado de hortalizas. Zaragoza: Acribia, 1992. p. 139-173.

BRASIL. Ministério da Saúde. Anvisa. Resolução no 362, de 29 de julho de 1999. Diário Oficial da União (DOU), 22 nov. 1999. Disponível em: <http://Www.anvisa.gov.br/legish 'resol/17_99rdc.htm'> Acesso em: 20 jul. 2004.

CAMARGO, R. Tecnologia dos produtos agropecuários: alimentos. São Paulo: Nobel, 1984. 298 p.

CHAIMSOHN, F. P. Cultivo de pupunha e produção de palmito. Viçosa: Aprenda Fácil, 2000. 121 p.

CHAIMSOHN, F. P. Desenvolvimento de pupunha (Bactris gasipaes Kunth) cultivada para palmito em diferentes regiões do Paraná. Boletim Técnico do Instituto Agronômico do Paraná, Londrina, n. 67, p. 1-54, 2002.

FELLOWS, P. Tecnología del procesado de los alimentos: principios y práticas. Zaragoza: Acribia, 1994. 549 p.

FERREIRA, V. L. P.; GRANER, M.; BOVI, M. L. A.; DRAETTA, I. S.; PASCHOALINO, J. E.; SHIROSE, I. Comparação entre os palmitos de Guilielma gasipaes Bailey (pupunha) e Euterpe edulis Mart. (juçara): II. avaliações físicas e químicas. Coletânea do Instituto de Tecnologia de Alimentos (ITAL), Campinas, v. 12, n. 1, p. 273-282, 1982.

FRANCO, B. D. G. M.; LANDGRAF, M. Microrganismos patogênicos de importância em alimentos. In: FRANCO, B. D. G. M.; LANDGRAF, M. Microbiologia dos alimentos. São Paulo: Atheneu, 1996. p. 33-41.

GAVA, A. J. Princípios de tecnologia de alimentos. Rio de Janeiro: Nobel, 1978. 284 p.

INSTITUTO ADOLFO LUTZ. Normas analíticas do Instituto Adolfo Lutz. São Paulo, 1985. 459 p.

INSTITUTO AGRONÔMICO DO PARANÁ. Curso sobre cultivo, processamento e comercialização de palmito de pupunha. Londrina, 2001. (Circular técnica, 117).

KAPP, E. A.; PINHEIRO, J. L.; RAUPP, D. S.; CHAIMSOHN, F. P. Tempo de preservação de tolete de palmito pupunha (Bactris gasipaes) minimamente processado e armazenado sob refrigeração. Publicatio UEPG Ciências Exatas e da Terra, Ciências Agronômicas, Ponta Grossa, v. 9, n. 3, p. 51-57, 2003.

LINDEN, G.; LORIENT, D. Bioquímica agroindustrial. Zaragoza: Acribia, 1996. 428 p.

MONTES, A. L. Microbiologia de los alimentos: curso teorico y practico. São Paulo: Ed. Resenha Universitária, $1977.576 \mathrm{p}$.

PASCHOALINO, J. E. Processamento de hortaliças. Campinas: ITAL, 1994. (Manual técnico, 4).

PASCHOALINO, J. E. Hortaliças acidificadas em conserva: riscos e cuidados. Informativo Fruthotec, Campinas, v. 3, n. 2, 1997.

PELCZAR, M.; REID, R.; CHAN, E. C. S. Microbiologia. São Paulo: McGraw-Hill do Brasil, 1981. v. 2, 1071 p.

RAUPP, D. S. Higiene e sanidade do produto palmito. In: ENCONTRO PARANAENSE SOBRE PALMITOS CULTIVADOS: O AGRONEGÓCIO PUPUNHA E PALMEIRA REAL, 1., 2002, Pontal do Paraná. Anais... Colombo: Embrapa-Florestas, 2002. p. 10-11.

RAUPP, D. S.; CHAIMSOHN, F. P. O envase de palmito de pupunha em vidro. In: KULCHETESCKI, L.; CAMISON, F. P.; GARDINGO, J. R. Palmito Pupunha (Bactris gasipaes Kunth): a espécie, cultura, manejo agronômico, usos e processamentos. [S.1.]: UEPG, 2001. p. 105-118.

RIEDEL, G. Controle sanitário dos alimentos. São Paulo: Atheneu, 1992. 320 p.

SOARES, A. G. Palmito de pupunha: alternativas de processamento. Horticultura Brasileira, Brasília, v. 15, p. 198-199, 1997. Suplemento.

TONET, R. M.; FERREIRA, L. G. S.; OTOBONI, J. L. M. A cultura da pupunha. Campinas: CATI, 1999. (Boletim técnico, 237).

ZAPATA, M. M.; QUAST, D. G. Curvas de titulação do palmito-doce (Euterpe edulis Mart.). Coletânea do Instituto de Tecnologia de Alimentos (ITAL), Campinas, v. 6, p. 167$187,1975$. 